\title{
Disclosure of same-sex practices and experiences of healthcare stigma among cisgender men who have sex with men in five sub-Saharan African countries
}

John Mark Wiginton ${ }^{1 *}$, Sarah M. Murray², Ohemaa Poku², Jura Augustinavicius², Kevon-Mark Phillip Jackman², Jeremy Kane ${ }^{3}$, Serge C. Billong ${ }^{4}$, Daouda Diouf ${ }^{5}$, Ibrahima Ba ${ }^{5}$, Tampose Mothopeng ${ }^{6}$, lliassou Mfochive Njindam,8, Gnilane Turpin $^{7,8}$, Ubald Tamoufe ${ }^{8}$, Bhekie Sithole ${ }^{9}$, Maria Zlotorzynska ${ }^{10}$, Travis H. Sanchez ${ }^{10}$ and Stefan D. Baral ${ }^{7}$

\begin{abstract}
Background: For men who have sex with men (MSM) across sub-Saharan Africa (SSA), disclosure of same-sex practices to family and healthcare workers (HCWs) can facilitate access to HIV prevention services and support, but can also lead to experiences of stigma.

Methods: We performed mixed-effects regressions on pooled data from MSM in Cameroon, Senegal, Côte d'Ivoire, Lesotho, and eSwatini to assess associations between disclosure and sexual behavior stigma in healthcare contexts; we used logistic regressions to analyze country-specific data.

Results: Compared to participants who had not disclosed to either family or HCWs, those who had disclosed only to family were more likely to have been gossiped about by HCWs ( $\mathrm{aOR}=1.70, \mathrm{Cl}=1.18,2.45)$; the association between having disclosed to family and having felt mistreated in a health center approached, but did not achieve, statistical significance $(\mathrm{aOR}=1.56, \mathrm{Cl}=0.94,2.59)$. Those who had disclosed only to HCWs were more likely to have feared to seek health services $(\mathrm{aOR}=1.60, \mathrm{Cl}=1.14,2.25)$, avoided health services $(\mathrm{aOR}=1.74, \mathrm{Cl}=1.22,2.50)$, and felt mistreated in a health center $(\mathrm{aOR}=2.62, \mathrm{Cl}=1.43,4.81)$. Those who had disclosed to both were more likely to have feared to seek health services ( $\mathrm{aOR}=1.71, \mathrm{Cl}=1.16,2.52)$, avoided health services $(\mathrm{aOR}=1.59, \mathrm{Cl}=1.04,2.42)$, been gossiped about by HCWs $(\mathrm{aOR}=3.78, \mathrm{Cl}=2.38,5.99)$, and felt mistreated in a health center $(\mathrm{aOR}=3.39, \mathrm{Cl}=$ 1.86, 6.20). Country-specific analyses suggested that data from Cameroon drove several of these associations.
\end{abstract}

Conclusions: Research to determine the factors driving disclosure's differential effect on healthcare stigma across contexts is needed. Ultimately, supportive environments enabling safe disclosure is critical to understanding HIVacquisition risks and informing differentiated HIV-prevention, treatment, and testing services for MSM across SSA.

Keywords: Disclosure, Healthcare stigma, Men who have sex with men, Sub-Saharan Africa

\footnotetext{
* Correspondence: jwigint2@jhmi.edu

1 Department of Health, Behavior \& Society, Johns Hopkins University

Bloomberg School of Public Health, 624 N Broadway Street, Baltimore, MD

21205, USA

Full list of author information is available at the end of the article
}

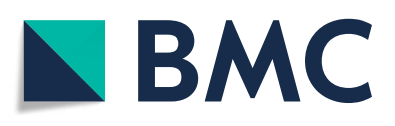

(c) The Author(s). 2021, corrected publication 2021. Open Access This article is licensed under a Creative Commons Attribution 4.0 International License, which permits use, sharing, adaptation, distribution and reproduction in any medium or format, as long as you give appropriate credit to the original author(s) and the source, provide a link to the Creative Commons licence, and indicate if changes were made. The images or other third party material in this article are included in the article's Creative Commons licence, unless indicated otherwise in a credit line to the material. If material is not included in the article's Creative Commons licence and your intended use is not permitted by statutory regulation or exceeds the permitted use, you will need to obtain permission directly from the copyright holder. To view a copy of this licence, visit http://creativecommons.org/ licenses/by/4.0/. The Creative Commons Public Domain Dedication waiver (http://creativecommons.org/publicdomain/zero/1. 0/) applies to the data made available in this article, unless otherwise stated in a credit line to the data. 


\section{Background}

Globally, men who have sex with men (MSM) continue to bear a disproportionate burden of HIV [1]. This burden is in part driven and exacerbated by intersecting social stigmas related to both same-sex practices and HIV [2-4]. These stigmas are especially severe in settings in the 36 countries across sub-Saharan Africa (SSA) that criminalize same-sex practices [4-7]. Sexual behavior stigma undermines HIV prevention efforts, including HIV testing, uptake of and adherence to HIV prevention and treatment medication, and engagement in other sexual health services $[8,9]$.

One mechanism through which sexual behavior stigma impedes HIV prevention is enacted healthcare stigma, which involves healthcare workers' (HCWs) overt discrimination (e.g., denial of services) and mistreatment (e.g., verbal harassment, gossip) of MSM due to engagement in same-sex practices $[10,11]$. Experiences of enacted healthcare stigma can disrupt HIV service utilization and dissuade MSM from future help-seeking [12, 13]. Sexual behavior stigma may further constrain healthcare access through perceived or anticipated healthcare stigma, which includes the perception, expectation, or fear of current or future mistreatment by HCWs due to one's engagement in same-sex practices [14-16]. Such stigma can lead to missed opportunities for preventive screening and other services, thereby increasing risk for HIV and sexually transmitted infections [17-19].

Experiences of sexual behavior stigma among MSM in healthcare contexts have often been linked to disclosure of engagement in same-sex practices to HCWs [7, 9 2023]. The relationship between healthcare stigma and disclosure of same-sex practices to family has been less researched, perhaps due to the more indirect, complex pathways between the two. Scholarship has linked family disclosure to difficulties accessing health services for MSM in SSA [24], possibly reflecting the loss of social and financial support from family following disclosure, which may explain why disclosure by MSM to family has also been linked to economic independence [23, 25]. Alternatively, the link between family disclosure and difficulties accessing healthcare could be due to perceived or anticipated healthcare stigma stemming from family disclosure experiences. That is, negative reactions from family following disclosure may prime MSM to anticipate or perceive similar reactions from HCWs, avoiding disclosure to HCWs or avoiding healthcare encounters altogether [23, 26]. Positive reactions from family following disclosure could also lead to healthcare stigma. MSM who feel validated and supported by their family may be less concerned with concealing their sexuality in other contexts, proudly disclosing their same-sex practices to HCWs or displaying gender nonconforming mannerisms that may be perceived as homosexual, incurring enacted stigma as a result [23, 27]. While nondisclosure could perhaps be protective against some of the impacts of sexual behavior stigma [28-30], it could also preclude MSM from reaping disclosure's benefits. Research has repeatedly established links between disclosure of same-sex practices by MSM to HCWs and receipt or utilization of appropriate HIV care and other sexual health services [31-33]. Similarly, disclosure to family may yield acceptance and affirmative social support, facilitate disclosure to HCWs, promote engagement in sexual health services, and support the adoption of positive health behaviors [30, 34-39].

Given the complex roles of disclosure across healthcare and family contexts, there remains a need to better understand how and the extent to which different disclosures are related to stigma within healthcare settings. We therefore assessed patterns of disclosure of same-sex practices to family and HCWs by cisgender MSM in five sub-Saharan African countries (Cameroon, Senegal, Côte d'Ivoire, Lesotho, eSwatini) and examined how such patterns may be differentially linked to multiple forms of healthcare stigma.

\section{Methods}

This study involved secondary analysis of cross-sectional data collected from cisgender MSM between 2013 and 2016 using respondent-driven sampling (RDS) (Cameroon, Senegal, eSwatini, Côte d'Ivoire) or snowball sampling (Lesotho). In the countries where RDS was used, 3-16 seeds selected by/from community-based organizations recruited participants across 12-20 waves of recruitment [4, 40-45]. Given that the lived experience of being transgender or nonbinary is distinct from that of being cisgender, we excluded data from gender minorities in this study, which require separate analyses focused on intersectional stigmas $[44,46]$. Cisgender men age $\geq 18$ years reporting past-year anal sex with a man completed a face-to-face survey assessing sexual behavior stigma, sexuality disclosure, HIV diagnosis and treatment, and sociodemographic and other characteristics. Participants received US\$2-\$6 for their time and travel costs, and for each eligible RDS recruit. Studies were conducted in compliance with local ethical standards and received the approval of local ethics committees and Johns Hopkins University institutional review board. This secondary analysis of deidentified data received exemption from Johns Hopkins University institutional review board. Detailed data collection methods have been described elsewhere [4, 40-45].

\section{Measures}

Outcome variables were four yes/no indicators of sexual behavior stigma related to the healthcare system: (1)"Have you ever felt afraid to go to healthcare services 
because you worry someone may learn you have sex with men?" (2) "Have you ever avoided going to healthcare services because you worry someone may learn you have sex with men?" (3) Have you ever heard healthcare providers gossiping about you (talking about you) because you have sex with men?" (4) "Have you ever felt that you were not treated well in a health center because someone knew that you have sex with men?" [4, 33, 40, 47, 48]. Items $1-2$ assessed anticipated healthcare stigma, and items 3-4 assessed enacted and perceived healthcare stigma. The exposure variable, disclosure of same-sex practices, was assessed using two yes/no items that asked if participants had ever told a (1) HCW or (2) family member about their engagement in same-sex practices.

Control variables included sociodemographic and other characteristics. Age was measured in years. Education was dichotomized into having completed secondary school or beyond versus not having finished secondary school. A dummy variable was created to indicate country of residence, and income quintiles were created based on within-country income comparisons. Selfreported HIV status was measured with three response options: positive, negative, and unknown. Depression, which was included due to its links to healthcare stigma and sexual behavior disclosure among MSM in other sub-Saharan African contexts [12, 19, 28, 30], was measured with the Patient Health Questionnaire-9 $[49,50]$. This questionnaire assessed the frequency of nine depressive symptoms within the previous two weeks. Response options included "not at all" (0), "several days" (1), "more than half the days" (2), and "nearly every day" (3). Items were summed, with a score of $\geq 5$ indicative of at least mild depression [51].

\section{Analysis}

Data from all countries were pooled and analyzed as crude data; RDS-adjusted weighting was not applied across countries as individuals did not represent a single network, violating a key assumption of RDS [52]. Frequency of endorsement and missingness for each stigma and disclosure item was assessed [15]. Descriptive statistics were calculated for sociodemographic and other variables, and chi-square tests (for categorical variables) and the Wilcoxon rank-sum test (for age) were used to compare differences in these variables by endorsement of each healthcare stigma item. Mixed-effects logistic regression models with a random effect for country were constructed to examine associations between healthcare stigma and disclosure. Initially, associations between each dichotomous disclosure variable and each healthcare stigma outcome variable were examined, with product terms (disclosure to a $\mathrm{HCW}^{*}$ disclosure to a family member) being incorporated to assess multiplicative interaction effects between both types of disclosure and healthcare stigma. However, as none of the interaction terms were significant in the pooled analysis and were excluded, we instead combined responses to create a four-level categorical variable: (1) have not disclosed to a $\mathrm{HCW}$ or family member (reference); (2) have disclosed to a family member only; (3) have disclosed to a HCW only; (4) have disclosed to both a family member and HCW.

In building the mixed-effect regression models, we first created empty models to assess the presence of significant clustering of each healthcare stigma outcome within countries. Next, the categorical disclosure variable was added to each empty model to examine bivariate associations with each healthcare stigma variable. Multivariable models were subsequently constructed, with the inclusion of control variables. In addition, separate logistic regression analyses were performed on selected country-specific data. Coefficients were exponentiated to generate odds ratios, and Wald tests, with significance set at $\alpha=0.05$, and 95\% confidence intervals were calculated and examined. Model results represent valid sample estimates but may not represent population-level estimates given the lack of RDSadjustment. Procedures were performed in Stata Version 15 [53].

\section{Results}

The total cross-country datasets included data from 4405 participants, 3284 of whom reported being a cisgender man and eligible for inclusion in this analysis; 244 participants were missing data on key variables and were excluded, resulting in a complete-case analysis sample size of $N=3040$. Most participants were from Côte d'Ivoire or Cameroon, followed by Lesotho, eSwatini, and Senegal (Table 1). Median age was 24 years, over three-quarters reported high school or less education, and $>40 \%$ reported income in the lowest quintile. Roughly one in six participants had clinically significant depressive symptoms. Approximately 6\% (172/3040) reported positive HIV status, and 25\% (758/3040) reported unknown HIV status.

Overall, 67.9\% (2064/3040) of participants had not disclosed their same-sex practices to a family member or HCW, 18.6\% (566/3040) had disclosed to a family member only, $8.0 \%(243 / 3040)$ had disclosed to a HCW only, and 5.5\% (167/3040) had disclosed to both. Disclosure to neither a family member nor a HCW was highest in Cameroon and lowest in Lesotho. Disclosure to a family member only was highest in Lesotho and lowest in Senegal. Disclosure to a HCW only was highest in Senegal and lowest in Cameroon. Disclosure to both was highest in eSwatini and lowest in Senegal (Fig. 1). Nearly one in five participants (539/3040) reported having felt 
Table 1 Characteristics of MSM in SSA, 2014-2016 ( $N=3040)$

\begin{tabular}{|c|c|}
\hline Country, n (\%) & \\
\hline Côte d'Ivoire & $947(31.2)$ \\
\hline Cameroon & $838(27.6)$ \\
\hline Lesotho & $476(15.7)$ \\
\hline eSwatini & $393(12.9)$ \\
\hline Senegal & $386(12.7)$ \\
\hline \multicolumn{2}{|l|}{ Age in years } \\
\hline Mean (SD) & $24.5(5.1)$ \\
\hline Median (IQR) & $24(21-27)$ \\
\hline \multicolumn{2}{|l|}{ Education, n (\%) } \\
\hline$\leq$ High school & $2386(78.5)$ \\
\hline$>$ High school & $654(21.5)$ \\
\hline \multicolumn{2}{|l|}{ Income, n (\%) } \\
\hline Quintile 1 & $1263(41.5)$ \\
\hline Quintile 2 & $460(15.1)$ \\
\hline Quintile 3 & $206(6.8)$ \\
\hline Quintile 4 & $627(20.6)$ \\
\hline Quintile 5 & $484(15.9)$ \\
\hline \multicolumn{2}{|l|}{ HIV status, n (\%) } \\
\hline Negative & $2110(69.4)$ \\
\hline Positive & $172(5.7)$ \\
\hline Unknown & $758(24.9)$ \\
\hline \multicolumn{2}{|l|}{ Depression, n (\%) } \\
\hline Yes & $490(16.1)$ \\
\hline No & $2550(83.9)$ \\
\hline \multicolumn{2}{|l|}{ Disclosure of same-sex practices } \\
\hline Not to a family member or a HCW, n (\%) & $2064(67.9)$ \\
\hline To a family member only, n (\%) & $566(18.6)$ \\
\hline To a HCW only, n (\%) & $243(8.0)$ \\
\hline To a family member and a HCW, n (\%) & $167(5.5)$ \\
\hline
\end{tabular}

MSM, men who have sex with men; SSA, sub-Saharan Africa; SD, standard deviation; IQR, interquartile range; HIV, human immunodeficiency virus; HCW healthcare worker

afraid to seek health services, while one in seven (436/ 3040) reported having avoided health services due to worry over someone learning they have sex with men. Seven percent of participants (211/3040) reported hearing healthcare providers gossip about them because they have sex with men, and 4\% (120/3040) reported having felt mistreated in a health center because they have sex with men (Table 2).

Compared to participants who had not disclosed to either a family member or HCW, those who had disclosed only to a family member were significantly more likely to have been gossiped about by healthcare providers; the association between having disclosed only to a family member and having felt mistreated in a health center

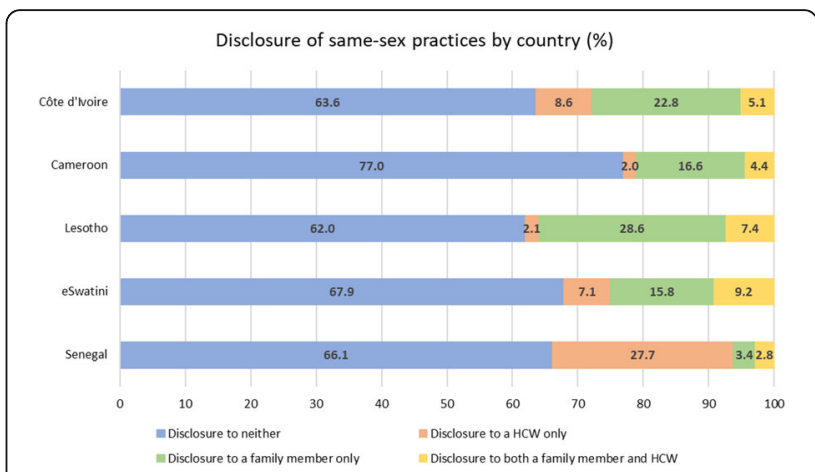

Fig. 1 Disclosure of same-sex practices among MSM in SSA (by country), 2014-2016 ( $N=3040)$. MSM, men who have sex with men; SSA, sub-Saharan Africa; HCW, healthcare worker

approached, but did not achieve, statistical significance. Those who had disclosed only to a HCW were significantly more likely to have feared to seek health services, avoided health services, and felt mistreated in a health center. Those who had disclosed to both were significantly more likely to have feared to seek health services, avoided health services, been gossiped about by healthcare providers, and felt mistreated in a health center (Table 3).

As residual variance due to country-level differences was significant and ranged from $5.3-8.6 \%$ across adjusted models (Table 3 ), we analyzed country-level data. However, exploratory analyses revealed small cell values of both disclosure and healthcare stigma experiences in most countries, with particularly small (and occasionally empty) cells in Lesotho and Senegal. We therefore restricted country-level regression analyses to Côte d'Ivoire and Cameroon, as each made up roughly $30 \%$ of the overall sample size. Analysis of Côte d'Ivoire data found some significant adjusted associations, which were positive and confined to perceived and enacted healthcare stigma (Table 4). Re-running the pooled country multilevel model without Côte d'Ivoire resulted in few changes compared to the pooled country multilevel model with Côte d'Ivoire included (see Additional Table 1).

We repeated this process with Cameroon, with slightly more significant and several trending adjusted associations emerging between disclosure and stigma in country-specific analyses. In the Cameroon data, compared to participants who had not disclosed to either a family member or HCW, those who had disclosed only to a family member were significantly more likely to have felt mistreated in a health center; the association between having disclosed only to a family member and having been gossiped about by healthcare providers approached, but did not achieve, statistical 
Table 2 Differences in sociodemographic and other characteristics by healthcare stigma among MSM in SSA, 2014-2016 (N=3040)

\begin{tabular}{|c|c|c|c|c|c|c|c|c|}
\hline & \multicolumn{2}{|c|}{ Afraid to seek health services } & \multicolumn{2}{|c|}{$\begin{array}{l}\text { Avoided seeking health } \\
\text { services }\end{array}$} & \multicolumn{2}{|c|}{$\begin{array}{l}\text { Gossiped about by } \\
\text { healthcare providers }\end{array}$} & \multicolumn{2}{|c|}{$\begin{array}{l}\text { Felt mistreated in a health } \\
\text { center }\end{array}$} \\
\hline & $\begin{array}{l}\text { Yes }(n=539 \\
17.7 \%)\end{array}$ & $\begin{array}{l}\text { No }(n=2501, \\
82.3 \%)\end{array}$ & $\begin{array}{l}\text { Yes }(n=436 \\
14.3 \%)\end{array}$ & $\begin{array}{l}\text { No }(n=2604, \\
85.7 \%)\end{array}$ & $\begin{array}{l}\text { Yes }(n=211, \\
6.9 \%)\end{array}$ & $\begin{array}{l}\text { No }(n=2829 \text {, } \\
93.1 \%)\end{array}$ & $\begin{array}{l}\text { Yes }(n=120, \\
3.9 \%)\end{array}$ & $\begin{array}{l}\text { No }(n=2920 \\
96.1 \%)\end{array}$ \\
\hline \multicolumn{9}{|l|}{ Country, n (\%) } \\
\hline Côte d'lvoire & $196(36.4)$ & $751(30.0)$ & $127(29.1)$ & $820(31.5)$ & $81(38.4)$ & $866(30.6)$ & $24(20.0)$ & $923(31.6)$ \\
\hline Cameroon & $82(15.2)$ & $756(30.2)$ & $79(18.1)$ & $759(29.2)$ & $40(19.0)$ & $798(28.2)$ & $25(20.8)$ & $813(27.8)$ \\
\hline Lesotho & $51(9.5)$ & $425(17.0)$ & $35(8.0)$ & $441(16.9)$ & $15(7.1)$ & $461(16.3)$ & $16(13.3)$ & $460(15.8)$ \\
\hline eSwatini & $144(26.7)$ & $249(10.0)$ & $138(31.7)$ & $255(9.8)$ & $57(27.0)$ & $336(11.9)$ & $45(37.5)$ & $348(11.9)$ \\
\hline Senegal & $66(12.2)$ & $320(12.8)$ & $57(13.1)$ & $329(12.6)$ & $18(8.5)$ & $368(13.0)$ & $10(8.3)$ & $376(12.9)$ \\
\hline $\begin{array}{l}\text { Chi-square } p \text { - } \\
\text { value }\end{array}$ & $<0.001$ & & $<0.001$ & & $<0.001$ & & $<0.001$ & \\
\hline \multicolumn{9}{|l|}{ Age in years } \\
\hline Mean (SD) & $24.4(4.9)$ & $24.5(5.3)$ & $24.7(4.7)$ & $24.5(5.3)$ & $25.1(5.3)$ & $24.4(5.2)$ & $25.4(5.5)$ & $24.5(5.2)$ \\
\hline Median (IQR) & $24(21-27)$ & $23(21-27)$ & $24(21-27)$ & $23(21-27)$ & $24(21-28)$ & $23(21-27)$ & $24(22-28)$ & $23(21-27)$ \\
\hline $\begin{array}{l}\text { Wilcoxon } p \text { - } \\
\text { value }\end{array}$ & 0.067 & & $<0.010$ & & $<0.010$ & & $<0.05$ & \\
\hline \multicolumn{9}{|l|}{ Education, n (\%) } \\
\hline$\leq$ High school & $386(71.6)$ & $2000(80.0)$ & $314(72.0)$ & $2072(79.6)$ & 151 (71.6) & $2235(79.0)$ & $90(75.0)$ & $2296(78.6)$ \\
\hline > High school & $153(28.4)$ & $501(20.0)$ & $122(28.0)$ & $532(20.4)$ & $60(28.4)$ & $594(21.0)$ & $30(25.0)$ & $624(21.4)$ \\
\hline $\begin{array}{l}\text { Chi-square } \mathrm{p}- \\
\text { value }\end{array}$ & $<0.001$ & & $<0.001$ & & $<0.050$ & & 0.343 & \\
\hline \multicolumn{9}{|l|}{ Income, n (\%) } \\
\hline Quintile 1 & $230(42.7)$ & $1033(41.3)$ & $162(37.2)$ & $1101(42.3)$ & $81(38.4)$ & $1182(41.8)$ & $31(25.8)$ & $1232(42.2)$ \\
\hline Quintile 2 & $66(12.2)$ & 394 (15.8) & 59 (13.5) & $401(15.4)$ & $32(15.2)$ & $428(15.1)$ & $17(14.2)$ & $443(15.2)$ \\
\hline Quintile 3 & $50(9.3)$ & $156(6.2)$ & $44(10.1)$ & $162(6.2)$ & $19(9.0)$ & $187(6.6)$ & $20(16.7)$ & $186(6.4)$ \\
\hline Quintile 4 & 105 (19.5) & $522(20.9)$ & 85 (19.5) & $542(20.8)$ & $41(19.4)$ & $586(20.7)$ & $31(25.8)$ & $596(20.4)$ \\
\hline Quintile 5 & $88(16.3)$ & $396(15.8)$ & $86(19.7)$ & $398(15.3)$ & $38(18.0)$ & $446(15.8)$ & $21(17.5)$ & $463(15.9)$ \\
\hline $\begin{array}{l}\text { Chi-square } p \text { - } \\
\text { value }\end{array}$ & $<0.05$ & & $<0.010$ & & 0.561 & & $<0.001$ & \\
\hline \multicolumn{9}{|l|}{ HIV status, n (\%) } \\
\hline Negative & $363(67.4)$ & $1747(69.9)$ & $294(67.4)$ & $1816(69.7)$ & 149 (70.6) & 1961 (69.3) & $78(65.0)$ & 2032 (69.6) \\
\hline Positive & $39(7.2)$ & $133(5.3)$ & $28(6.4)$ & $144(5.5)$ & $18(8.5)$ & $154(5.4)$ & $12(10.0)$ & $160(5.5)$ \\
\hline Unknown & $137(25.4)$ & $621(24.8)$ & $114(26.2)$ & $644(24.7)$ & $44(20.9)$ & $714(25.2)$ & $30(25.0)$ & $728(24.9)$ \\
\hline $\begin{array}{l}\text { Chi-square } p \text { - } \\
\text { value }\end{array}$ & 0.188 & & 0.574 & & $<0.088$ & & 0.105 & \\
\hline \multicolumn{9}{|l|}{ Depression, n (\%) } \\
\hline Yes & $161(29.9)$ & $329(13.2)$ & $149(34.2)$ & $341(13.1)$ & 71 (33.6) & 419 (14.8) & $51(42.5)$ & $439(15.0)$ \\
\hline No & $378(70.1)$ & $2172(86.8)$ & $287(65.8)$ & $2263(86.9)$ & $140(66.4)$ & $2410(85.2)$ & $69(57.5)$ & $2481(85.0)$ \\
\hline $\begin{array}{l}\text { Chi-square } p \text { - } \\
\text { value }\end{array}$ & $<0.001$ & & $<0.001$ & & $<0.001$ & & $<0.001$ & \\
\hline
\end{tabular}

MSM, men who have sex with men; SSA, sub-Saharan Africa; SD, standard deviation; IQR, interquartile range; HIV, human immunodeficiency virus

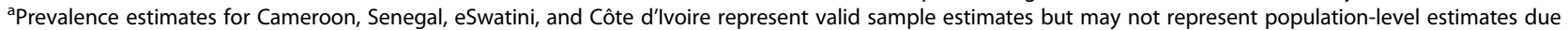
to lack of adjustment for respondent-driven sampling

significance. Those who disclosed only to a HCW were significantly more likely to have feared to seek health services and avoided health services; the association between having disclosed only to a HCW and having been gossiped about by healthcare providers approached, but did not achieve, statistical significance. Those who had disclosed to both were significantly more likely to have feared to seek health services; the association between having disclosed to both and having avoided health services and having felt mistreated in a health center 
Table 3 Associations between disclosure of same-sex practices and healthcare stigma among MSM in SSA (pooled), 2014-2016 $(\mathrm{N}=3040)$

\begin{tabular}{|c|c|c|c|c|c|c|c|c|c|c|c|c|}
\hline & \multicolumn{3}{|c|}{$\begin{array}{l}\text { Afraid to seek health } \\
\text { services }\end{array}$} & \multicolumn{3}{|c|}{$\begin{array}{l}\text { Avoided health } \\
\text { services }\end{array}$} & \multicolumn{3}{|c|}{$\begin{array}{l}\text { Gossiped about by } \\
\text { healthcare providers }\end{array}$} & \multicolumn{3}{|c|}{$\begin{array}{l}\text { Felt mistreated in a } \\
\text { health center }\end{array}$} \\
\hline & Empty & $\begin{array}{l}\text { OR } \\
(95 \% \mathrm{Cl})\end{array}$ & $\begin{array}{l}\mathrm{aOR} \\
(95 \% \mathrm{Cl})\end{array}$ & Empty & $\begin{array}{l}\text { OR } \\
(95 \% \mathrm{Cl})\end{array}$ & $\begin{array}{l}\text { aOR } \\
(95 \% \mathrm{Cl})\end{array}$ & Empty & $\begin{array}{l}\text { OR } \\
(95 \% \mathrm{Cl})\end{array}$ & $\begin{array}{l}\mathrm{aOR} \\
(95 \% \mathrm{Cl})\end{array}$ & Empty & $\begin{array}{l}\text { OR } \\
(95 \% \mathrm{Cl})\end{array}$ & $\begin{array}{l}\text { aOR } \\
(95 \% \mathrm{Cl})\end{array}$ \\
\hline \multicolumn{13}{|l|}{ Fixed effects } \\
\hline \multicolumn{13}{|c|}{ Same-sex practices disclosure } \\
\hline $\begin{array}{l}\text { Not to a FM } \\
\text { or a HCW } \\
\text { (ref) }\end{array}$ & - & 1.00 & 1.00 & - & 1.00 & 1.00 & - & 1.00 & 1.00 & - & 1.00 & 1.00 \\
\hline To a FM only & - & $\begin{array}{l}1.04 \\
(0.80, \\
1.35)\end{array}$ & $\begin{array}{l}1.04(0.80 \\
1.35)\end{array}$ & - & $\begin{array}{l}1.02 \\
(0.77, \\
1.36)\end{array}$ & $\begin{array}{l}1.04(0.77 \\
1.39)\end{array}$ & - & $\begin{array}{l}1.66^{* *} \\
(1.16 \\
2.37)\end{array}$ & $\begin{array}{l}1.70^{* *} \\
(1.18, \\
2.45)\end{array}$ & - & $\begin{array}{l}1.42 \\
(0.86, \\
2.33)\end{array}$ & $\begin{array}{l}1.56 \\
\sim(0.94 \\
2.59)\end{array}$ \\
\hline $\begin{array}{l}\text { To a HCW } \\
\text { only }\end{array}$ & - & $\begin{array}{l}1.64^{* *} \\
(1.18 \\
2.27)\end{array}$ & $\begin{array}{l}1.60^{* *} \\
(1.14, \\
2.25)\end{array}$ & - & $\begin{array}{l}1.76^{* *} \\
(1.24 \\
2.50)\end{array}$ & $\begin{array}{l}1.74^{* * *} \\
(1.22 \\
2.50)\end{array}$ & - & $\begin{array}{l}1.45 \\
(0.86, \\
2.45)\end{array}$ & $\begin{array}{l}1.32(0.77 \\
2.25)\end{array}$ & - & $\begin{array}{l}2.79^{* *} \\
(1.55 \\
5.03)\end{array}$ & $\begin{array}{l}2.62^{* *} \\
(1.43 \\
4.81)\end{array}$ \\
\hline $\begin{array}{l}\text { To a FM and } \\
\text { a HCW }\end{array}$ & - & $\begin{array}{l}1.93^{* *} \\
(1.33 \\
2.79)\end{array}$ & $\begin{array}{l}1.71^{* *} \\
(1.16, \\
2.52)\end{array}$ & - & $\begin{array}{l}1.77^{* *} \\
(1.18 \\
2.65)\end{array}$ & $\begin{array}{l}1.59 * \\
(1.04, \\
2.42)\end{array}$ & - & $\begin{array}{l}4.14^{* * *} \\
(2.67 \\
6.40)\end{array}$ & $\begin{array}{l}3.78^{* * *} \\
(2.38 \\
5.99)\end{array}$ & - & $\begin{array}{l}4.44^{* * *} \\
(1.95 \\
6.05)\end{array}$ & $\begin{array}{l}3.39 * * * \\
(1.86 \\
6.20)\end{array}$ \\
\hline \multicolumn{13}{|l|}{ Random effect } \\
\hline $\begin{array}{l}\text { Country-level } \\
\text { variance (SE) }\end{array}$ & $\begin{array}{l}0.35^{* * *} \\
(0.23)\end{array}$ & $\begin{array}{l}0.34^{* * *} \\
(0.22)\end{array}$ & $\begin{array}{l}0.28^{* * *} \\
(0.19)\end{array}$ & $\begin{array}{l}0.42^{* * *} \\
(0.28)\end{array}$ & $\begin{array}{l}0.40^{* * *} \\
(0.27)\end{array}$ & $\begin{array}{l}0.31^{* * *} \\
(0.21)\end{array}$ & $\begin{array}{l}0.31^{* * *} \\
(0.22)\end{array}$ & $\begin{array}{l}0.30^{* * *} \\
(0.21)\end{array}$ & $\begin{array}{l}0.26^{* * *} \\
(0.20)\end{array}$ & $\begin{array}{l}0.35^{* * *} \\
(0.25)\end{array}$ & $\begin{array}{l}0.28^{* * *} \\
(0.20)\end{array}$ & $\begin{array}{l}0.19^{* *} \\
(0.15)\end{array}$ \\
\hline \multicolumn{13}{|c|}{ Model characteristics } \\
\hline $\begin{array}{l}\text { Intra-class } \\
\text { correlation }\end{array}$ & $9.7 \%$ & $9.3 \%$ & $7.9 \%$ & $11.4 \%$ & $10.9 \%$ & $8.6 \%$ & $8.5 \%$ & $8.3 \%$ & $7.5 \%$ & $9.7 \%$ & $9.9 \%$ & $5.3 \%$ \\
\hline
\end{tabular}

$\sim p<0.10 ;{ }^{*} p<0.05 ;{ }^{* *} p<0.01 ;{ }^{* * *} p<0.001$

MSM, men who have sex with men; SSA, sub-Saharan Africa; OR, odds ratio; aOR, adjusted odds ratio; Cl, confidence interval; FM, family member; HCW, healthcare worker; SE, standard error

Controlling for age, education, income, HIV status, and depression

Table 4 Associations between disclosure of same-sex practices and healthcare stigma among MSM in SSA (by country), 2014-2016 $(\mathrm{N}=3040)$

\begin{tabular}{|c|c|c|c|c|c|c|c|c|}
\hline & \multicolumn{2}{|c|}{$\begin{array}{l}\text { Afraid to seek } \\
\text { health services }\end{array}$} & \multicolumn{2}{|c|}{$\begin{array}{l}\text { Avoided seeking } \\
\text { health services }\end{array}$} & \multicolumn{2}{|c|}{$\begin{array}{l}\text { Gossiped about by } \\
\text { healthcare providers }\end{array}$} & \multicolumn{2}{|c|}{$\begin{array}{l}\text { Felt mistreated in a } \\
\text { health center }\end{array}$} \\
\hline & $\begin{array}{l}\text { OR } \\
(95 \% \mathrm{Cl})\end{array}$ & $\begin{array}{l}\text { aOR } \\
(95 \% \mathrm{Cl})\end{array}$ & $\begin{array}{l}\text { OR } \\
(95 \% \mathrm{Cl})\end{array}$ & $\begin{array}{l}\text { aOR } \\
(95 \% \mathrm{Cl})\end{array}$ & $\begin{array}{l}\text { OR } \\
(95 \% \mathrm{Cl})\end{array}$ & $\begin{array}{l}\text { aOR } \\
(95 \% \mathrm{Cl})\end{array}$ & $\begin{array}{l}\text { OR } \\
(95 \% \mathrm{Cl})\end{array}$ & $\begin{array}{l}\text { aOR } \\
(95 \% \mathrm{Cl})\end{array}$ \\
\hline \multicolumn{9}{|l|}{ Same-sex practices disclosure } \\
\hline Not to a FM or a HCW (ref) & 1.00 & 1.00 & 1.00 & 1.00 & 1.00 & 1.00 & 1.00 & 1.00 \\
\hline \multicolumn{9}{|l|}{ Côte d'Ivoire } \\
\hline To a FM only & $\begin{array}{l}0.99 \\
(0.67,1.47)\end{array}$ & $\begin{array}{l}0.95 \\
(0.88,2.64)\end{array}$ & $\begin{array}{l}1.23 \\
(0.78,1.94)\end{array}$ & $\begin{array}{l}1.19 \\
(0.74,1.89)\end{array}$ & $\begin{array}{l}1.84^{*} \\
(1.09,3.11)\end{array}$ & $\begin{array}{l}1.79^{*} \\
(1.05,3.04)\end{array}$ & $\begin{array}{l}1.80 \\
(0.69,4.70)\end{array}$ & $\begin{array}{l}1.75 \\
(0.66,4.63)\end{array}$ \\
\hline To a HCW only & $\begin{array}{l}1.73^{*} \\
(1.03,2.90)\end{array}$ & $\begin{array}{l}1.52 \\
(0.88,2.64)\end{array}$ & $\begin{array}{l}1.96^{*} \\
(1.09,3.52)\end{array}$ & $\begin{array}{l}1.75 \\
\sim(0.93,3.30)\end{array}$ & $\begin{array}{l}0.73 \\
(0.25,2.10)\end{array}$ & $\begin{array}{l}0.58 \\
(0.20,1.74)\end{array}$ & $\begin{array}{l}0.67 \\
(0.09,5.27)\end{array}$ & $\begin{array}{l}0.31 \\
(0.03,3.15)\end{array}$ \\
\hline To a family and a HCW & $\begin{array}{l}1.37 \\
(0.69,2.71)\end{array}$ & $\begin{array}{l}1.25 \\
(0.61,2.55)\end{array}$ & $\begin{array}{l}1.26 \\
(0.54,2.91)\end{array}$ & $\begin{array}{l}1.19 \\
(0.49,2.88)\end{array}$ & $\begin{array}{l}3.68^{* * *} \\
(2.26,9.70)\end{array}$ & $\begin{array}{l}4.16 * * * \\
(1.93,8.99)\end{array}$ & $\begin{array}{l}6.25^{* *} \\
(2.08,18.80)\end{array}$ & $\begin{array}{l}3.96^{*} \\
(1.17,13.39)\end{array}$ \\
\hline \multicolumn{9}{|l|}{ Cameroon } \\
\hline To a FM only & $\begin{array}{l}1.73 \\
\sim(0.96,3.12)\end{array}$ & $\begin{array}{l}1.55 \\
(0.85,2.83)\end{array}$ & $\begin{array}{l}1.18 \\
(0.62,2.23)\end{array}$ & $\begin{array}{l}1.06 \\
(0.55,2.04)\end{array}$ & $\begin{array}{l}1.87 \\
(0.85,4.14)\end{array}$ & $\begin{array}{l}2.07 \\
\sim(0.91,4.71)\end{array}$ & $\begin{array}{l}3.22^{*} \\
(1.29,8.04)\end{array}$ & $\begin{array}{l}3.53^{*} \\
(1.36,9.19)\end{array}$ \\
\hline To a HCW only & $\begin{array}{l}6.78^{* * *} \\
(2.40,19.14)\end{array}$ & $\begin{array}{l}5.59 * * \\
(1.88,16.57)\end{array}$ & $\begin{array}{l}6.22^{* *} \\
(2.21,17.50)\end{array}$ & $\begin{array}{l}4.84^{* *} \\
(1.64,14.28)\end{array}$ & $\begin{array}{l}5.80^{* *} \\
(1.56,21.58)\end{array}$ & $\begin{array}{l}3.62 \\
\sim(0.89,14.65)\end{array}$ & $\begin{array}{l}7.03^{*} \\
(1.45,34.22)\end{array}$ & $\begin{array}{l}4.40 \\
(0.74,26.15)\end{array}$ \\
\hline To a FM and a HCW & $\begin{array}{l}5.26^{* * *} \\
(2.45,11.29)\end{array}$ & $\begin{array}{l}4.37 * * \\
(1.87,10.19)\end{array}$ & $\begin{array}{l}3.15^{* *} \\
(1.37,7.23)\end{array}$ & $\begin{array}{l}2.25 \\
\sim(0.90,5.61)\end{array}$ & $\begin{array}{l}4.23^{* *} \\
(1.51,11.84)\end{array}$ & $\begin{array}{l}2.30 \\
(0.73,7.31)\end{array}$ & $\begin{array}{l}4.65^{*} \\
(1.25,17.27)\end{array}$ & $\begin{array}{l}3.78 \\
\sim(0.83,17.30)\end{array}$ \\
\hline
\end{tabular}

$\sim \mathrm{p}<0.10 ;{ }^{*} \mathrm{p}<0.05 ;{ }^{* *} \mathrm{p}<0.01$; ${ }^{* * *} \mathrm{p}<0.001$; significant, adjusted associations are also bolded

MSM, men who have sex with men; SSA, sub-Saharan Africa; OR, odds ratio; aOR, adjusted odds ratio; $\mathrm{Cl}$, confidence interval; FM, family member; HCW,

healthcare worker

Controlling for age, education, income, HIV status, and depression 
approached, but did not achieve, statistical significance (Table 4). Re-running the pooled country multilevel model without Cameroon revealed fewer significant associations compared to the pooled model that included data from Cameroon; these associations were positive and somewhat similar to those observed in the Cote D'Ivoire-only analysis (see Additional Table 2).

\section{Discussion}

We assessed patterns of disclosure of same-sex practices and examined their associations with four experiences of healthcare stigma among cisgender MSM in Côte d'Ivoire, Cameroon, Lesotho, eSwatini, and Senegal. Notably, a large majority of participants in each country had not disclosed their engagement in same-sex practices to either a family member or $\mathrm{HCW}$. In pooled-country analyses, we detected several positive associations between disclosure to a family member, $\mathrm{HCW}$, or both and each form of healthcare stigma. Country-specific analyses revealed that several of the associations observed between stigma and disclosure were driven by data from Cameroon, where non-disclosure was highest.

Among disclosure patterns, disclosure only to a family member was by far the most common in all countries except Senegal. This finding, coupled with the otherwise non-disclosing trend, showcases the rarity of disclosing one's same-sex practices to HCWs. Such non-disclosure to HCWs necessarily constrains one's access to and utilization of HIV-preventive and other sexual health services $[8,9]$. Interventions facilitating safe, private disclosure that also train healthcare providers to respond affirmatively without stigmatization are urgently needed for MSM in these countries. Disclosure only to a family member was linked to perceived and enacted healthcare stigma, illustrating how disclosure to family is complexly linked to experiences of stigma in other contexts even when disclosure has not explicitly occurred within that context. Negative reactions to disclosures of same-sex practices from family may prime men to perceive potentially benign interactions in healthcare or other contexts negatively and ascribe those interactions to their sexuality, even if the men have not actually made such disclosures, particularly if men have high rejection sensitivities [54]. This could account for the link between disclosure and the nonspecific feeling of having felt mistreated in a health center. MSM who have disclosed to family only may simultaneously hold a less-concealable sexual or other identity or status, increasing their vulnerability to enacted stigma in other contexts, despite having not disclosed their same-sex practices in those contexts [23], or they may be marginalized in some other way that we did not measure that resulted in enacted healthcare stigma.

Similarly, disclosure only to a $\mathrm{HCW}$ was linked to perceived and enacted healthcare stigma. For MSM who have disclosed their same-sex practices to a HCW, the nonspecific feeling of having been mistreated could arise from a range of experiences, including overt enacted stigma, such as gossip or denial of services; more subtle forms of enacted stigma, such as microaggressions [55, 56]; or relatively benign interactions for MSM with high rejection sensitivities [54]. Sexuality-based stigma may also intersect with HIV stigma, increasing the likelihood of stigmatization [12, 57]. That disclosure only to a HCW was linked to enacted stigma reflects prior research that has consistently shown an association between disclosure and a range of enacted healthcare stigmas, including denial of services and verbal harassment, adding to the range of obstacles with which MSM must contend when seeking appropriate sexual health services [7, 9, 20-23].

Disclosure only to a HCW was also linked to having feared and avoided healthcare services (anticipated stigmas), but only in Cameroon. Same-sex practices remain highly stigmatized and criminalized in Cameroon, making disclosure of same-sex practices particularly risky there [58-62], though there have been increased efforts to address these issues in recent years [63]. MSM who have experienced or perceived negative reactions to their disclosure of same-sex practices or who are aware of the potential for these reactions may fear and avoid situations where such reactions are possible, including healthcare situations [54, 64], as has been demonstrated in other subSaharan African countries [13, 26, 31, 65, 66]. That Cameroon appeared to drive these associations could be indicative of a more unique context of stigmatization relative to the other countries, requiring a more tailored approach to stigma-mitigation. Multilevel stigma-mitigation interventions, such as a recently implemented three-tiered integrated stigma mitigation intervention in Senegal [67], could be particularly impactful in Cameroon.

We did not find a synergistic effect of disclosure to both a family member and HCW on experiences of healthcare stigma, possibly due to insufficient power to detect such an effect, with relatively small sample sizes in some countries, as well as small cell values for experiences of both disclosure and stigma from low endorsement in some cases. This lack of synergism could also indicate that family and healthcare stigmas operate independently, with family stigma not detracting from welcoming, affirming healthcare experiences. Alternatively, this lack of synergism could reflect a certain resilience and resistance to experiences of healthcare stigma. For MSM who have disclosed or are out about their samesex practices in multiple contexts, sexuality may hold more salience and valence for their sense of self and identity, which may be protective against healthcare stigma [68]. Similarly, the overall low endorsement of healthcare stigmas - regardless of disclosure status - is 
significant. Being able to avoid stigmatizing experiences in healthcare settings, particularly in regions where same-sex practices are stigmatized and even criminalized, may reflect a certain degree of resilience among participants, undergirded by social cohesion and community support $[46,69,70]$.

Findings should be considered in light of several limitations. We only assessed verbal disclosure of same-sex practices. Recent research has shown that behavioral disclosure by MSM, conveyed via gender nonconforming mannerisms, may incur more stigma than verbal disclosure of same-sex practices [27]. Future research that assesses both disclosures may be useful for better understanding how each is differentially or synergistically associated with stigma. An intersectional approach will be appropriate in such research, as other stigmas such as those based on class and income, HIV status, or other attributes, which may require disclosures of their own - may intersect with sexuality- and gender-based stigmas. For example, MSM who engage in same-sex practices, display gender-nonconforming mannerisms, and are of low socioeconomic status may be stigmatized in ways that MSM of high socioeconomic status (but who are otherwise similar) are not, as the latter may have access to better sexual health services and providers, or may simply be treated better due to having higher income. Second, the directionality of the associations cannot be established due to the cross-sectional nature of the data. While it is perhaps more plausible that disclosure led to anticipating or experiencing stigma in healthcare, as was hypothesized and modeled in the present study, the opposite is also possible. For example, participants who disclosed their same-sex practices to a $\mathrm{HCW}$ and/or family may have feared or avoided healthcare prior to ever having made any disclosure about their sexuality simply because they have an awareness of the pervasive social stigma associated with same-sex practices in the context in which they live. Likewise, participants who were or felt mistreated by a $\mathrm{HCW}$ may have presumed it was due to assumptions about their undisclosed sexuality (or it may have been due to gender nonconforming behaviors) [27]; such a scenario may have prompted participants to confront the individual(s) they believed had stigmatized them, leading to their verbal disclosure of same-sex practices.

Additionally, social desirability bias may have influenced participant responses, as surveys were administered in-person in environments where same-sex practices are highly stigmatized. Fourth, the rarity of some country-specific disclosure patterns and experiences of healthcare stigma, combined with relatively small sample sizes, resulted in low precision of several parameter estimates and wide confidence intervals, potentially impacting the extent to which associations could be detected in some contexts. Lastly, respondentdriven and snowball sampling methods were used to recruit participants, limiting the generalizability of the findings. Moreover, the variation in disclosure and healthcare stigma experiences across countries observed in this study suggests there may be heterogeneous findings and unmeasured confounders (e.g., enforcement of laws targeting sexual minorities, social norms) from countries which were not studied, further complicating the extent to which generalizations can be made.

These findings underscore the need for not only the eradication of all forms of stigma and discrimination in healthcare contexts, but the presence of intentional affirmation of minority sexualities and marginalized persons. Supportive environments that enable safe disclosure are critical for providing appropriate sexual health services to MSM and maintaining their engagement in sexual health service utilization. For many MSM in countries across SSA, disclosure of same-sex practices lies at a critical intersection of tradeoffs, potentially leading to improved social support and sexual healthcare services (e.g., HIV pre-exposure prophylaxis access; improved counseling tailored to same-sex practices/partnerships) but also to stigmatization and victimization. Preventing sexuality-based stigma in healthcare settings requires fundamental change to heteronormative societal structures that perpetuate harmful norms and stereotypes, and maintain policies that stigmatize sexual minority men. Ultimately, advancing the HIV response in SSA and around the world necessitates multilevel interventions to mitigate community, institutional, and interpersonal-level stigma; foster enabling environments for disclosure of same-sex practices; and facilitate access to broader social and extrafamilial supports for MSM who have experienced stigma.

\section{Abbreviations}

MSM: men who have sex with men; HIV: human immunodeficiency virus; SSA: sub-Saharan Africa; HCW: healthcare worker; RDS: respondent-driven sampling

\section{Supplementary Information}

The online version contains supplementary material available at https://doi. org/10.1186/s12889-021-12151-3.

\section{Additional file 1.}

\section{Acknowledgments}

Not applicable.

Authors' contributions

JMW conceived and conducted the analysis, as well as drafted the manuscript. SM and SDB supervised the analysis and writing, and regularly reviewed the manuscript and provided feedback. OP contributed to the introduction, and JA reviewed the manuscript and provided feedback. KMPJ and JK contributed to early conceptualizations of the study, and reviewed the manuscript and suggested edits. SCB, DD, IB, TM, IMN, GT, UT, and BS were our in-country partners who helped lead data collection, as well as 
reviewed the manuscript and provided feedback and suggested edits. MZ and THS reviewed the manuscript, provided feedback, and suggested edits. All authors read and approved the final manuscript.

\section{Funding}

This study was funded by National Institutes of Health (R01MH110358). This study received ethical approval from within-country ethics committees and institutional review boards, and from a US-based academic institution's institutional review board.

\section{Availability of data and materials}

The datasets analyzed during the current study are not publicly available due to the sensitivity of the information collected but are available from the senior author (SDB) on reasonable request.

\section{Declarations}

\section{Ethics approval and consent to participate}

Studies were conducted in compliance with local ethical standards, received the approval of local ethics committees and a US-based academic institutional review board, and were conducted with participants' informed consent. This secondary analysis of deidentified data received exemption from the Johns Hopkins University Institutional Review Board.

\section{Consent for publication}

Not applicable.

\section{Competing interests}

The authors declare that they have no competing interests.

\section{Author details}

'Department of Health, Behavior \& Society, Johns Hopkins University Bloomberg School of Public Health, 624 N Broadway Street, Baltimore, MD 21205, USA. ²Department of Mental Health, Johns Hopkins University Bloomberg School of Public Health, Baltimore, MD, USA. ${ }^{3}$ Department of Epidemiology, Columbia University Mailman School of Public Health, New York City, NY, USA. ${ }^{4}$ Department of Public Health, Faculty of Medicine and Biomedical Sciences, University of Yaoundé I, Yaoundé, Cameroon; Central Technical Group, National AIDS Control Committee, Yaoundé, Cameroon. ${ }^{5}$ Enda Santé, Dakar, Senegal. ${ }^{6}$ The People's Matrix, Maseru, Lesotho. ${ }^{7}$ Center for Public Health \& Human Rights, Department of Epidemiology, Johns Hopkins University Bloomberg School of Public Health, Baltimore, MD, USA. ${ }^{8}$ Metabiota, Yaounde, Cameroon, Johns Hopkins Cameroon Program, Yaounde, Cameroon. ${ }^{9}$ MbabaneFHI 360, MbabaneeSwatini. ${ }^{10}$ Department of Epidemiology, Emory University Rollins School of Public Health, Atlanta, GA, USA.

\section{Received: 29 July 2021 Accepted: 28 October 2021}

\section{Published online: 03 December 2021}

\section{References}

1. Joint United Nations Program on HIV/AIDS (UNAIDS). UNAIDS data 2019. 2019.

2. Alvy LM, McKirnan D, Du Bois SN, Jones K, Ritchie N, Fingerhut D. Health Care Disparities and Behavioral Health Among Men Who Have Sex with Men. J Gay Lesbian Soc Serv 2011 10/01;23(4):507-522.

3. Stoloff K, Joska JA, Feast D, De Swardt G, Hugo J, Struthers H, et al. A description of common mental disorders in men who have sex with men (MSM) referred for assessment and intervention at an MSM clinic in Cape Town, South Africa. AIDS Behav 2013 May;17 Suppl 1:S77-81.

4. Stahlman S, Sanchez TH, Sullivan PS, Ketende S, Lyons C, Charurat ME, et al. the prevalence of sexual behavior stigma affecting gay men and other men who have sex with men across sub-Saharan Africa and in the United States. JMIR public health Surveill 2016 Jul 26;2(2):e35.

5. Ross MW, Nyoni J, Larsson M, Mbwambo J, Agardh A, Kashiha J, et al. Health care in a homophobic climate: the SPEND model for providing sexual health services to men who have sex with men where their health and human rights are compromised. Glob Health Action. 2015 Mar 17;8(1): 26096. https://doi.org/10.3402/gha.v8.26096.

6. Amnesty International. Making love a crime: criminalization onf same-sex conduct in sub-Saharan Africa. 2013.
7. Fay H, Baral SD, Trapence G, Motimedi F, Umar E, lipinge S, et al. Stigma, health care access, and HIV knowledge among men who have sex with men in Malawi, Namibia, and Botswana. AIDS Behav. 2011 Aug;15(6):108897. https://doi.org/10.1007/s10461-010-9861-2.

8. Altman D, Aggleton P, Williams M, Kong T, Reddy V, Harrad D, et al. Men who have sex with men: stigma and discrimination. Lancet. $2012 \mathrm{Jul} 28$; 380(9839):439-45. https://doi.org/10.1016/S0140-6736(12)60920-9.

9. Beyrer C, Sullivan PS, Sanchez J, Dowdy D, Altman D, Trapence G, et al. A call to action for comprehensive HIV services for men who have sex with men. Lancet. 2012 Jul 28;380(9839):424-38. https://doi.org/10.1016/S01406736(12)61022-8.

10. Earnshaw VA, Chaudoir SR. From conceptualizing to measuring HIV stigma: a review of HIV stigma mechanism measures. AIDS Behav. 2009 Dec;13(6): 1160-77. https://doi.org/10.1007/s10461-009-9593-3.

11. Jacoby A. Felt versus enacted stigma: a concept revisited. Evidence from a study of people with epilepsy in remission. Soc Sci Med. 1994 Jan;38(2):26974. https://doi.org/10.1016/0277-9536(94)90396-4.

12. Kennedy CE, Baral SD, Fielding-Miller R, Adams D, Dludlu P, Sithole B, et al. "They are human beings, they are Swazi": intersecting stigmas and the positive health, dignity and prevention needs of HIV-positive men who have sex with men in Swaziland. J Int AIDS Soc 2013 Dec 2;16 Suppl 3(4Suppl 3):18749.

13. Lane T, Mogale T, Struthers H, McIntyre J, Kegeles SM. "they see you as a different thing": the experiences of men who have sex with men with healthcare workers in south African township communities. Sex Transm Infect. 2008 Nov;84(6):430-3. https://doi.org/10.1136/sti.2008.031567.

14. Fisher CB, Fried AL, Macapagal K, Mustanski B. Patient-provider communication barriers and facilitators to HIV and STI preventive Services for Adolescent MSM. AIDS Behav. 2018 Oct;22(10):3417-28. https://doi.org/1 0.1007/s10461-018-2081-X

15. Augustinavicius JL, Baral SD, Murray SM, Jackman K, Xue QL, Sanchez TH, et al. Characterizing cross-culturally relevant metrics of stigma among men who have sex with men across 8 sub-Saharan African countries and the United States. Am J Epidemiol. 2020 Jul 1;189(7):690-7. https://doi.org/10.1 093/aje/kwz270

16. Herek G, Chopp R, Strohl D. Sexual stigma: putting sexual minority health issues in context. In: Meyer I, Northridge M, editors. The health of sexual minorities: public health perspectives on lesbian, gay, bisexual, and transgender populations Boston. MA: Springer Science + Business Media; 2007. p. 171-208. https://doi.org/10.1007/978-0-387-31334-4_8.

17. Baral S, Burrell E, Scheibe A, Brown B, Beyrer C, Bekker LG. HIV risk and associations of HIV infection among men who have sex with men in periurban Cape Town, South Africa. BMC Public Health. 2011 Oct 5;11:7662458-11-766.

18. Kim HY, Grosso A, Ky-Zerbo O, Lougue M, Stahlman S, Samadoulougou C, et al. Stigma as a barrier to health care utilization among female sex workers and men who have sex with men in Burkina Faso. Ann Epidemiol. 2018 Jan;28(1):13-9. https://doi.org/10.1016/j.annepidem.2017.11.009.

19. Risher K, Adams D, Sithole B, Ketende S, Kennedy C, Mnisi Z, et al. Sexual stigma and discrimination as barriers to seeking appropriate healthcare among men who have sex with men in Swaziland. J Int AIDS Soc. 2013 Nov 13;16(3 Suppl 2):18715. https://doi.org/10.7448/IAS.16.3.18715.

20. Duby Z, Nkosi B, Scheibe A, Brown B, Bekker LG. 'Scared of going to the clinic': Contextualising healthcare access for men who have sex with men female sex workers and people who use drugs in two South African cities. South Afr J HIV Med. 2018 Jan 19;19(1):701.

21. Baral S, Trapence G, Motimedi F, Umar E, lipinge S, Dausab F, et al. HIV prevalence, risks for HIV infection, and human rights among men who have sex with men (MSM) in Malawi, Namibia, and Botswana. PLoS One. 2009; 4(3):e4997. https://doi.org/10.1371/journal.pone.0004997.

22. Zahn R, Grosso A, Scheibe A, Bekker LG, Ketende S, Dausab F, et al. Human rights violations among men who have sex with men in southern Africa: comparisons between legal contexts. PLoS One. 2016 Jan 14;11(1):e0147156. https://doi.org/10.1371/journal.pone.0147156.

23. Stahlman S, Bechtold K, Sweitzer S, Mothopeng T, Taruberekera N, Nkonyana J, et al. Sexual identity stigma and social support among men who have sex with men in Lesotho: a qualitative analysis. Reprod Health Matters. 2015 Nov;23(46):127-35. https://doi.org/10.1016/j.rhm.201 5.11.017.

24. Ruiseñor-Escudero H, Lyons C, Ketende S, Pitche V, Simplice A, Tchalla J, et al. Prevalence and factors associated to disclosure of same-sex practices 
to family members and health care workers among men who have sex with men in Togo. AIDS Care. 2019 Aug;31(8):1019-25. https://doi.org/10.1 080/09540121.2019.1612000.

25. White Y, Sandfort T, Morgan K, Carpenter K, Pierre R. Family relationships and sexual orientation disclosure to family by gay and bisexual men in Jamaica. Int J Sex Health. 2016;28(4):306-17. https://doi.org/10.1080/1931 7611.2016.1227015.

26. Kokogho A, Amusu S, Baral SD, Charurat ME, Adebajo S, Makanjuola O, et al. Disclosure of Same-Sex Sexual Practices to Family and Healthcare Providers by Men Who Have Sex with Men and Transgender Women in Nigeria. Arch Sex Behav 2020 Mar 19.

27. Ross MW, Kashiha J, Mgopa LR. Stigmatization of men who have sex with men in health care settings in East Africa is based more on perceived gender role-inappropriate mannerisms than having sex with men. Glob Health Action. 2020 Dec 31;13(1):1816526. https://doi.org/10.1080/1654971 6.2020 .1816526 .

28. Stahlman S, Grosso A, Ketende S, Pitche V, Kouanda S, Ceesay N, et al. Suicidal ideation among MSM in three west African countries: associations with stigma and social capital. Int J Soc Psychiatry. 2016 Sep;62(6):522-31. https://doi.org/10.1177/0020764016663969.

29. Ryan C, Huebner D, Diaz RM, Sanchez J. Family rejection as a predictor of negative health outcomes in white and Latino lesbian, gay, and bisexual young adults. Pediatrics. 2009 Jan;123(1):346-52. https://doi.org/10.1542/ peds.2007-3524

30. Gyamerah AO, Collier KL, Reddy V, Sandfort TGM. Sexuality disclosure among black south African MSM and responses by family. J Sex Res. 2019 Nov-Dec;56(9):1203-18. https://doi.org/10.1080/00224499.2018.1559917.

31. Schwartz SR, Nowak RG, Orazulike I, Keshinro B, Ake J, Kennedy S, et al. The immediate eff ect of the same-sex marriage prohibition act on stigma, discrimination, and engagement on HIV prevention and treatment services in men who have sex with men in Nigeria: analysis of prospective data from the TRUST cohort. Lancet HIV. 2015 Jul;2(7):e299-306. https://doi.org/1 0.1016/S2352-3018(15)00078-8.

32. Petroll $A E$, Mosack KE. Physician awareness of sexual orientation and preventive health recommendations to men who have sex with men. Sex Transm Dis. 2011 Jan;38(1):63-7. https://doi.org/10.1097/OLQ.0b013e3181ebd50f.

33. Lyons C, Stahlman S, Holland C, Ketende S, Van Lith L, Kochelani D, et al. Stigma and outness about sexual behaviors among cisgender men who have sex with men and transgender women in Eswatini: a latent class analysis. BMC Infect Dis. 2019 Mar 5;19(1):211-019-3711-2. https://doi.org/1 0.1186/s12879-019-3711-2.

34. Chambers LA, Rueda S, Baker DN, Wilson MG, Deutsch R, Raeifar E, et al. Stigma, HIV and health: a qualitative synthesis. BMC Public Health. 2015 Sep 3;15:848-015-2197-0.

35. Bouris A, Hill BJ. Exploring the mother-adolescent relationship as a promotive resource for sexual and gender minority youth. J Soc Issues. 2017 Sep;73(3):618-36. https://doi.org/10.1111/josi.12234

36. Simons LG, Sutton TE, Simons RL, Gibbons FX, Murry VM. Mechanisms that link parenting practices to Adolescents' risky sexual behavior: a test of six competing theories. J Youth Adolesc. 2016 Feb;45(2):255-70. https://doi. org/10.1007/s10964-015-0409-7.

37. Stupiansky NW, Liau A, Rosenberger J, Rosenthal SL, Tu W, Xiao S, et al. Young Men's disclosure of same sex behaviors to healthcare providers and the impact on health: results from a US National Sample of young men who have sex with men. AIDS Patient Care STDs. 2017 Aug;31(8):342-7. https://doi.org/10.1089/apc.2017.0011.

38. Henry E, Awondo P, Fugon L, Yomb Y, Spire B. Coming out of the Nkuta: disclosure of sexual orientation associated with reduced risk behavior among MSM in Cameroon. Arch Sex Behav. 2012 Jun;41(3):525-7. https:// doi.org/10.1007/s10508-012-9916-8.

39. Zhao Y, Ma Y, Chen R, Li F, Qin X, Hu Z. Non-disclosure of sexual orientation to parents associated with sexual risk behaviors among gay and bisexual MSM in China. AIDS Behav. 2016 Jan;20(1):193-203. https://doi.org/10.1007/ s10461-015-1135-6.

40. Poteat T, Ackerman B, Diouf D, Ceesay N, Mothopeng T, Odette KZ, et al. HIV prevalence and behavioral and psychosocial factors among transgender women and cisgender men who have sex with men in 8 African countries: a cross-sectional analysis. PLoS Med. 2017 Nov 7;14(11):e1002422. https:// doi.org/10.1371/journal.pmed.1002422.

41. Stahlman S, Grosso A, Ketende S, Mothopeng T, Taruberekera N, Nkonyana $J$, et al. Characteristics of men who have sex with men in southern Africa who seek sex online: a cross-sectional study. J Med Internet Res 2015 May 25;17(5):e129.

42. Rao A, Stahlman S, Hargreaves J, Weir S, Edwards J, Rice B, et al. Sampling Key Populations for HIV Surveillance: Results From Eight Cross-Sectional Studies Using Respondent-Driven Sampling and Venue-Based Snowball Sampling. JMIR Public Health Surveill 2017 Oct 20;3(4):e72.

43. Stahlman S, Johnston LG, Yah C, Ketende S, Maziya S, Trapence G, et al. Respondent-driven sampling as a recruitment method for men who have sex with men in southern sub-Saharan Africa: a cross-sectional analysis by wave. Sex Transm Infect. 2016 Jun;92(4):292-8. https://doi.org/10.1136/sextra ns-2015-052184.

44. Scheim A, Lyons C, Ezouatchi R, Liestman B, Drame F, Diouf D, et al. Sexual behavior stigma and depression among transgender women and cisgender men who have sex with men in Côte d'Ivoire. Ann Epidemiol. 2019 May;33: 79-83.e1.

45. Baral SD, Ketende S, Mnisi Z, Mabuza X, Grosso A, Sithole B, et al. A crosssectional assessment of the burden of HIV and associated individual- and structural-level characteristics among men who have sex with men in Swaziland. J Int AIDS Soc. 2013 Dec 2;16(Suppl 3(4Suppl 3)):18768.

46. Moran A, Scheim A, Lyons C, Liestman B, Drame F, Ketende S, et al. Characterizing social cohesion and gender identity as risk determinants of HIV among cisgender men who have sex with men and transgender women in Côte d'Ivoire. Ann Epidemiol. 2020 Feb;42:25-32. https://doi. org/10.1016/j.annepidem.2019.11.003.

47. Stahlman S, Liestman B, Ketende S, Kouanda S, Ky-Zerbo O, Lougue M, et al. Characterizing the HIV risks and potential pathways to HIV infection among transgender women in cote d'Ivoire, Togo and Burkina Faso. J Int AIDS Soc. 2016 Jul 17;19(3 Suppl 2):20774. https://doi.org/10.7448/IAS.19.3.20774.

48. Ulanja MB, Lyons C, Ketende S, Stahlman S, Diouf D, Kouamé A, et al. The relationship between depression and sexual health service utilization among men who have sex with men (MSM) in Côte d'Ivoire, West Africa. BMC Int Health Hum Rights. 2019 Mar 5;19(1):11-019-0186-6. https://doi. org/10.1186/s12914-019-0186-6.

49. Adewuya $\mathrm{AO}$, Ola BA, Afolabi OO. Validity of the patient health questionnaire (PHQ-9) as a screening tool for depression amongst Nigerian university students. J Affect Disord. 2006 Nov;96(1-2):89-93. https://doi. org/10.1016/j.jad.2006.05.021.

50. Pence BW, Gaynes BN, Atashili J, O'Donnell JK, Tayong G, Kats D, et al. Validity of an interviewer-administered patient health questionnaire-9 to screen for depression in HIV-infected patients in Cameroon. J Affect Disord. 2012 Dec 20;143(1-3):208-13. https://doi.org/10.1016/j.jad.2012.05.056.

51. Kroenke K, Spitzer RL, Williams JB. The PHQ-9: validity of a brief depression severity measure. J Gen Intern Med. 2001 Sep;16(9):606-13. https://doi.org/1 0.1046/j.1525-1497.2001.016009606.x.

52. Salganik MJ, Heckathorn DD. Sampling and Estimation in Hidden Populations Using Respondent-Driven Sampling. Sociological Methodology 2004 2021/10:34:193-239.

53. StatCorp LLC. Stata statistical software, release 15. 2017. College Station, Texas.

54. Feinstein BA. The rejection sensitivity model as a framework for understanding sexual minority mental health. Arch Sex Behav. 2019 Jul;8(7): 2247-58. https://doi.org/10.1007/s10508-019-1428-3.

55. Nadal KL. Issa M-, Leon J, Meterko V, Wideman M, Wong Y. sexual orientation microaggressions: "death by a thousand cuts" for lesbian, gay, and bisexual youth. J LGBT Youth. 2011;8(3):234-59. https://doi.org/10.1 080/19361653.2011.584204.

56. Francis DA, Reygan F. 'Let's See if it Won't Go Away by Itself.' LGBT Microaggressions among Teachers in South Africa. Education as Change 2016 09;20(3):180-201.

57. Friedland BA, Sprague L, Nyblade L, Baral SD, Pulerwitz J, Gottert A, et al. Measuring intersecting stigma among key populations living with HIV implementing the people living with HIV Stigma Index 2.0. J Int AIDS Soc 2018 Jul;21 Suppl 5(Suppl Suppl 5):e25131.

58. Bowring AL, Ketende S, Rao A, Mfochive Njindam I, Decker MR, Lyons C, et al. Characterising unmet HIV prevention and treatment needs among young female sex workers and young men who have sex with men in Cameroon: a cross-sectional analysis. Lancet Child Adolesc Health. 2019 Jul; 3(7):482-91. https://doi.org/10.1016/S2352-4642(19)30123-3.

59. Cange CW, LeBreton M, Billong S, Saylors K, Tamoufe U, Papworth E, et al. Influence of stigma and homophobia on mental health and on the uptake of HIV/sexually transmissible infection services for Cameroonian men who have sex with men. Sex Health. 2015 Aug;12(4):315-21. https://doi.org/10.1071/SH15001. 
60. Human Rights Watch. Cameroon: Same-sex relations bring attacks, arrests. 2010.

61. Human Rights Watch. Criminalizing identities: Rights abuses in Cameroon based on sexual orientation and gender identity. 2010.

62. Republic of Cameroon. Penal code (Law 65-LF-24, 12 November 1965 and Law 67-LF-1, 12 June 1967) Art 347 bis-Homosexuality.

63. The Global Fund to Fight AIDS, TB and Malaria. Baseline assessment -

Cameroon: Scaling up programs to reduce human rights-related barriers to HIV and TB services. 2018.

64. Caceres CF, Pecheny M, Frasca T, Rios RR. Review of legal frameworks and the situation of human rights related to sexual diversity in low and middle income countries; 2009.

65. Ntata PR, Muula AS, Siziya S. Socio-demographic characteristics and sexual health related attitudes and practices of men having sex with men in central and southern Malawi. Tanzan J Health Res. 2008 Jul;10(3):124-30. https://doi.org/10.4314/thrb.v10i3.14351.

66. Sharma A, Bukusi E, Gorbach P, Cohen CR, Muga C, Kwena Z, et al. Sexual identity and risk of HIV/STI among men who have sex with men in Nairobi. Sex Transm Dis. 2008 Apr;35(4):352-4. https://doi.org/10.1097/OLQ.0b013e31 815 e6320.

67. Lyons CE, Ketende S, Diouf D, Drame FM, Liestman B, Coly K, Ndour C, Turpin G, Mboup S, Diop K, Toure-Kane C, Castor D, Leye-Diouf N, Baral S Potential Impact of Integrated Stigma Mitigation Interventions in Improving HIV/AIDS Service Delivery and Uptake for Key Populations in Senegal. J Acquir Immune Defic Syndr 2017 Jan 1;74 Suppl 1(Suppl 1):S52-S59, https://doi.org/10.1097/QAl.0000000000001209.

68. Meyer $\mathbb{H}$. Prejudice, social stress, and mental health in lesbian, gay, and bisexual populations: conceptual issues and research evidence. Psychol Bull. 2003 Sep;129(5):674-97. https://doi.org/10.1037/0033-2909.129.5.674.

69. Stahlman S, Grosso A, Ketende S, Sweitzer S, Mothopeng T, Taruberekera N, et al. Depression and social stigma among MSM in Lesotho: implications for HIV and sexually transmitted infection prevention. AIDS Behav. 2015 Aug; 19(8):1460-9. https://doi.org/10.1007/s10461-015-1094-y.

70. Brown CA, Grosso AL, Adams D, Sithole B, Ketende S, Greene J, et al. Characterizing the individual, social, and structural determinants of condom use among men who have sex with men in Swaziland. AIDS Res Hum Retrovir. 2016 Jun;32(6):539-46. https://doi.org/10.1089/aid.2015.0240.

\section{Publisher's Note}

Springer Nature remains neutral with regard to jurisdictional claims in published maps and institutional affiliations.

Ready to submit your research? Choose BMC and benefit from:

- fast, convenient online submission

- thorough peer review by experienced researchers in your field

- rapid publication on acceptance

- support for research data, including large and complex data types

- gold Open Access which fosters wider collaboration and increased citations

- maximum visibility for your research: over $100 \mathrm{M}$ website views per year

At $\mathrm{BMC}$, research is always in progress.

Learn more biomedcentral.com/submissions 